\title{
Electrooptic effect in cholesteric blue phases with small positive dielectric anisotropy
}

\author{
B. M. Leon Fong and Jose A. Martin-Pereda
}

\begin{abstract}
An electrooptic effect in the blue phase of the cholesteric mixture S811- and the nematic mixture N5 is reported. To demonstrate this effect ac voltages $(f=1000 \mathrm{~Hz})$ between 0 and $150 \mathrm{~V}$ were applied. Wavelength shifts of $70 \mathrm{~nm}$ were obtained.
\end{abstract}

\section{Introduction}

In the past few years, there has been renewed interest in the properties of the so-called cholesteric blue phase (BP), which occurs in many cholesterol derivatives and chiral compounds in a narrow temperature range between isotropic and usual cholesteric (helicoidal) phases. ${ }^{1}$ Although this anomalous phase was first observed in 1906 by Lehmann, ${ }^{2}$ its main properties have not yet found concrete applications. In the middle of the Seventies an intensive study of these phases was undertaken. Several theoretical models have been proposed and several thermooptic effects have been reported..$^{3-7}$

As known from liquid crystal textbooks, ${ }^{8}$ the cholesteric mesophases formed by esters of cholesterol offer selective reflection of circularly polarized light in the ultraviolet region at temperatures close to the clearing point when they are pure. However, cooling down cholesteric esters only a few tenths of a kelvin below the clearing point results in bright colors appearing (mostly blue), which disappear on further cooling. These colors, as pointed out by Lehmann, belong to an optically isotropic modification. The name of blue phase has been used for them because of the striking light reflection effect. Different opinions have questioned whether BPs were thermodynamically stable phases or nothing but special textures of cholesterics. But after the work

B. M. Leon Fong is with Escuela Tecnica Superior, Ingenieros Industriales, Vigo, Spain, and Jose A. Martin-Pereda is with Escuela Tecnica Superior de Ingenieros de Telecommunicacion, Madrid28040, Spain.

Received 9 February 1985.

0003-6935/85/172842-04\$02.00/0.

(c) 1985 Optical Society of America. done by Armitage and Price in $1975,{ }^{9}$ the assumption of a stable phase existing between the isotropic liquid and the cholesteric state has been clearly proved. Several subsequent experiments have shown the existence of up to three different BPs (blue phase I, blue phase II, and blue fog).

Their practical applications still remain far from being in common use, due to their small temperature range of existence. In fact, the first studies with BPs showed a few tenths of a degree for their interval of existence. Recent studies have shown ${ }^{10,11}$ that BPs of mixtures can have temperature ranges of several degrees; therefore, there is some interest in evaluating the possibilities for applications of BPs in the electrooptic industry. Their use can be related to similar fields where cholesteric mesophases are being studied. In fact, the high rotatory power of cholesteric liquid crystals is interesting for practical uses when a suitable switching is available. Moreover, it is well known that an electric field is able to induce deformations on the cholesteric structure; hence, variations of the rotatory power vs the applied electric field are reported. These deformations modify the pitch of the cholesteric mesophase shifting the selective reflection band. Similar effects are expected to be found in the BP case.

A problem concerning BPs and their electrooptic properties is that theoretical explanation is difficult. This comes from the several theoretical models ${ }^{4,5,11}$ proposed concerning their geometric structure, among which the most probable seems to be the cubic, either simple or body centered. 4,5

In this paper we report an electrooptic effect in the blue phase of cholesteric mixtures. A theoretical approach is given in the light of the BP model proposed by Meiboom et al. ${ }^{4}$

\section{Experimental Methods}

Our cells were filled with a cholesteric mixture of S811- 


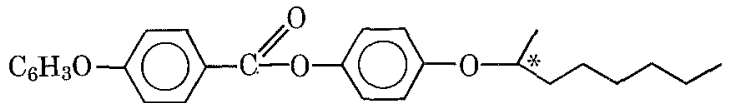

and the nematic mixture N5, both from Merck. N5 is a eutectic mixture of azoxy compounds. No further references are given by the manufacturers. A study of the BP phase diagram of these mixtures ${ }^{10}$ has shown that a sample with composition $31.35 \mathrm{wt}$. \% of S811- has the widest $\mathrm{BP}$ temperature range $(2 \mathrm{~K})$. Hence this composition was used for electrooptic measurements. The value of the dielectric anisotropy of N5 is $\Delta \varepsilon=-0.2$. The samples $w$ were contained in 23- $\mu \mathrm{m}$ glass cells.

The spectroscopic and electrooptic measurements were performed in a Cary 210 (Varian) spectrometer in transmission using left circularly polarized light. Taking into account that the samples studied are transparent for wavelengths $>420 \mathrm{~nm}$ (absorption edge of N5), it is possible to measure the reflection spectra through absorption data.

The sample cells were in a brass block, temperature controlled to an accuracy of $\pm 0.01^{\circ} \mathrm{C}$ using distilled water and an S-F3 Haake temperature regulator. The temperature was measured with a Chromel-Alumel thermoelement.

To demonstrate the electrooptic effect ac voltages $(f$ $=1000 \mathrm{~Hz}$ ) between 0 and $150 \mathrm{~V}$ were applied, while the reflection spectra were measured at a constant temperature. Higher voltages were not applied to avoid dielectric breakdown.

\section{Experimental Results}

The reflection spectra of the sample with $31.35 \mathrm{wt}$. \% S811- temperatures are shown in Fig. 1. These spectra were measured in one run successively coding from curves 1 to 14. Each reflection spectrum shows a sharp peak at a certain wavelength, $\lambda_{\max }$. This denotes a good surface alignment of the blue phase structure which can also be confirmed microscopically. The homogeneous texture of the BP II with quadratic symmetry is shown in Fig. 2.

As the temperature is lowered the selective reflection maximum, $\lambda_{\max }$, shifts continuously to higher wavelengths. At the BP II $\rightarrow$ BP I transition (curve 4 in Fig. 1), a weak second peak appears at a wavelength $60 \mathrm{~nm}$ higher. Under further cooling (curve 5) the BP II peak vanishes and the BP I peak grows as high as the former BP II one was. Further cooling results in a continuous displacement of the BP I- $\lambda_{\max }$ to the red side of the visible spectrum. It should be noted that, because our microscopic and spectroscopic measurements were both in the transmission mode, the clearing point could not be determined with accuracy due to the absorption edge of $\mathrm{N} 5$ at $420 \mathrm{~nm}$.

Figure 3 shows the reflective maxima position as a function of the temperature; the range where blue fog appears in the microscope is also shown. As can be seen, a variation of $2 \mathrm{~K}$ in the temperature gives a change from 630 to $470 \mathrm{~nm}$ with a sharp transition from 562 to $505 \mathrm{~nm}$ at $32.07^{\circ} \mathrm{C}$.

With the above maxima in mind, corresponding to both BPs, we have applied an electric field to see its

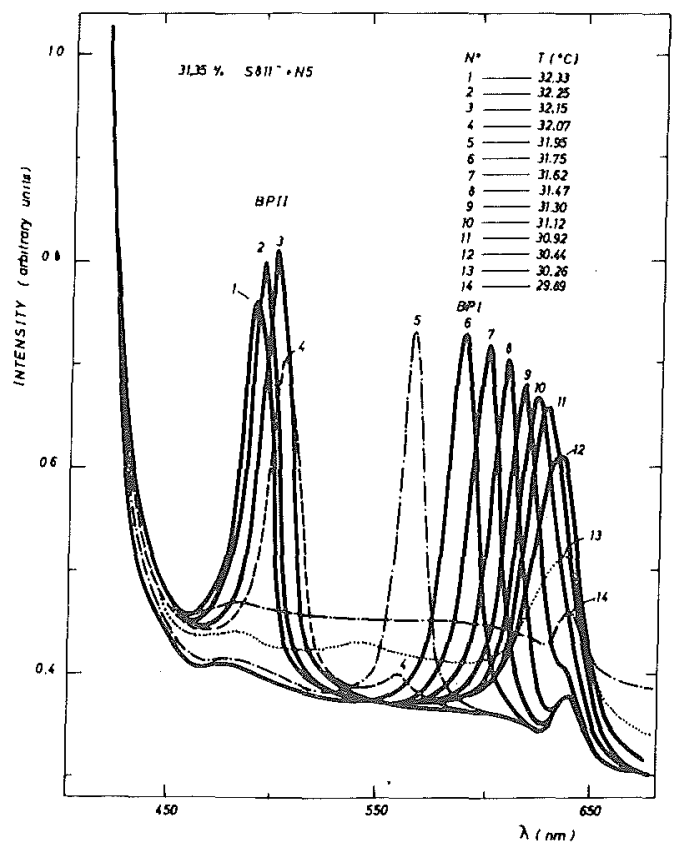

Fig. 1. Reflection spectra for different temperatures.

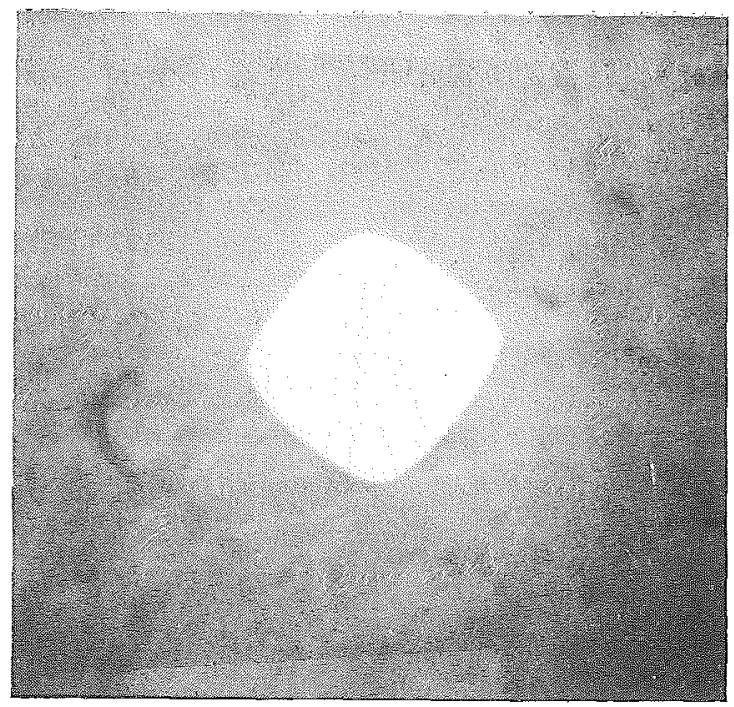

Fig. 2. Blue phase II quadratic domain.

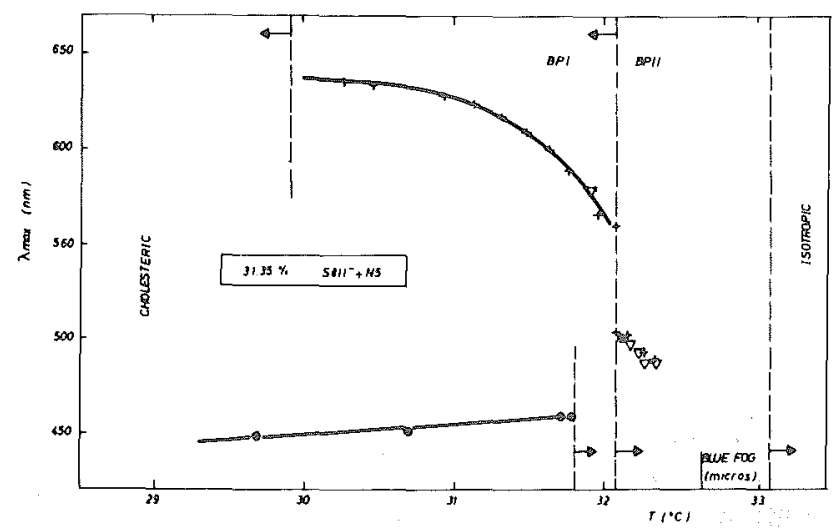

Fig. 3. Reflective maxima position as a function of the temperature. The different types of point belong to different measurement runs. 


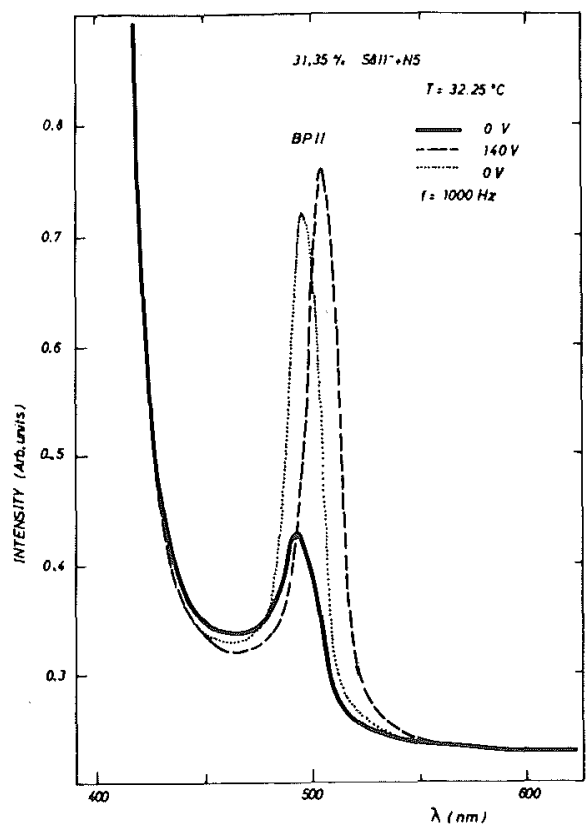

Fig. 4. Electrooptic effect of the BP II. The reflective maximum shifts to larger wavelengths by application of an electric field.

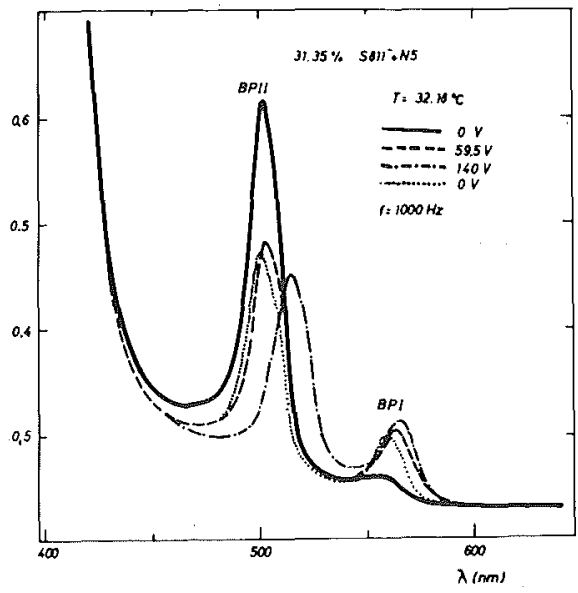

Fig. 5. Partial switching from BP II to BP I by application of an electric field.

influence on them. Our results are summarized in Figs. 4-7. The main results are:

(a) Electric fields applied to BP II shifts the maximum toward larger wavelengths but with few nanometers of displacement (Fig. 4). If $140 \mathrm{~V}(E=6.09 \times$ $10^{4} \mathrm{~V} / \mathrm{cm}$ ) are applied, a shift of $11 \mathrm{~nm}$ is obtained. The peak increases its intensity as a consequence of a better cell orientation with respect to the incident light. If the electric field is turned off, the maximum returns to the initial position but with higher intensity.

(b) If the initial state is close to the BP II $\rightarrow$ BP I transition (Fig. 5), a voltage of $59.5 \mathrm{~V}$ lowers the BP II peak to half of its value and raises the BP I peak.

At higher voltages both peaks shift to larger wavelengths. If the electric field is turned off, both peaks return to their original positions but, in the case of the BP II, its intensity is much lower than at the beginning.

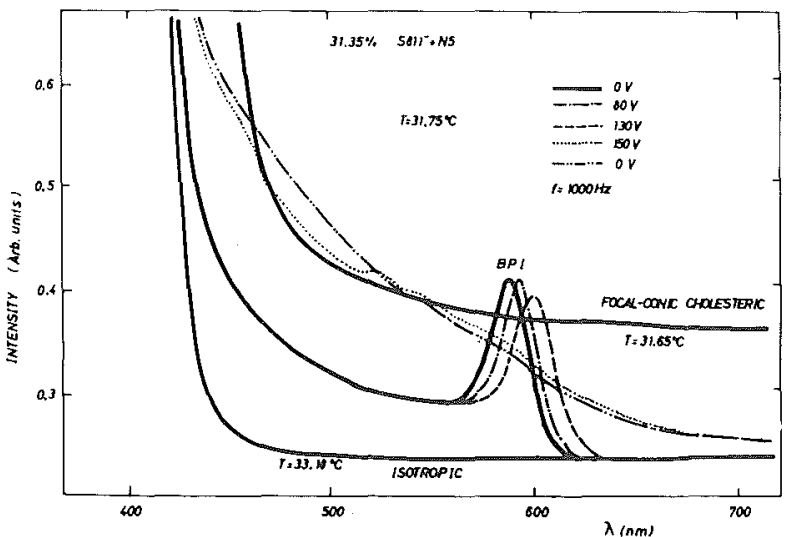

Fig. 6. Shift of the BP I maximum to a larger wavelength and in duction of the cholesteric phase by application of an electric field.

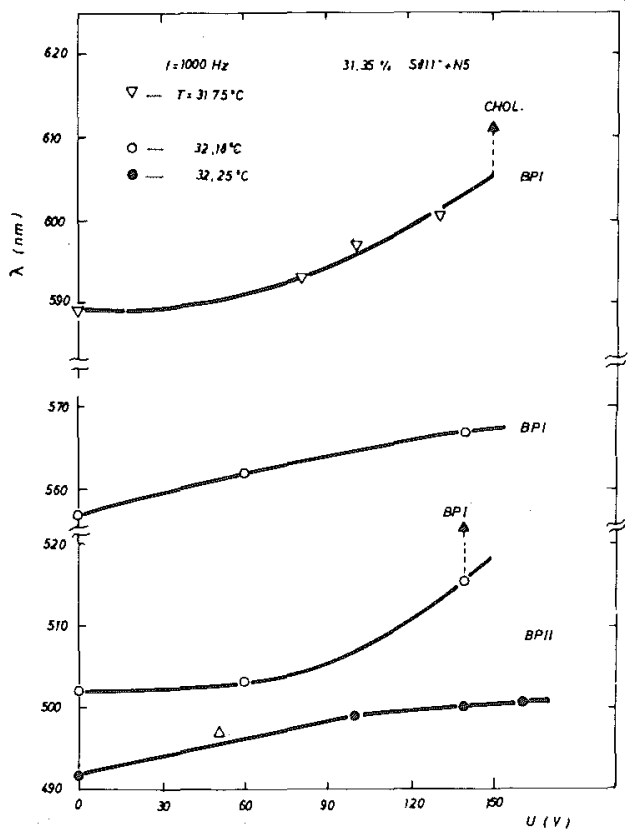

Fig. 7. Maxima positions as a function of the applied voltage for different temperatures.

On the contrary, the BP I has a higher peak. Presumably higher fields would eliminate the BP II.

At $31.96^{\circ} \mathrm{C}$, where just the $\mathrm{BP} \mathrm{I}$ is present, a transition from BP I to BP II was induced by $50.8 \mathrm{~V}$. Possibly this effect is caused not by the field but by dielectric heating.

(c) At lower temperatures (Fig. 6), electric fields shift the maximum toward larger wavelengths. At $150 \mathrm{~V}$ the maximum vanishes and the spectrum shows a cholesteric structure. A similar shape is obtained when the field is switched off.

The above facts are summarized in Fig. 7, where the maxima positions are represented as a function of the applied voltage and for different temperatures. As can be seen, every $\lambda_{\max }(\mathrm{V})$ curve shows a positive slope. Hence, electric fields give rise to similar effects in blue phases as the temperature decreases. ${ }^{11}$ 


\section{Theoretical Approach and Conclusions}

According to the blue phase model proposed by Meiboom et al. ${ }^{4}$ the blue phase is locally double twisted to satisfy chirality in two directions perpendicular to the molecular long axis. To make this double twisting compatible throughout the volume of the cholesteric it is necessary to introduce a (cubic) lattice of disclinations, which explains the isotropy of such phases. Such phenomena would only occur sufficiently close to the phase transition in the isotropic liquid where the energy to create defects or isotropic regions is not prohibitive. Thus, one could state that the BP is locally more isotropic than the normal cholesteric, in the sense that it twists around two directions instead of around one, respectively. Hence, the BP is thermodynamically a more disordered phase than the cholesteric. In fact; its temperature range of existence is higher. Accordingly, the BP II is more disordered than the BP I.

Assuming that an external applied electric field has an ordering effect on these phases independent of the sign of the dielectric anisotropy, it is anticipated that increasing the field strength should have a similar effect on the phase sequence to that of lowering the temperature. This intuitive relationship is confirmed by the results reported here.

To summarize: an electrooptic effect in blue phases with low dielectric anisotropy has been reported. In our case, the mixture employed had a value of +0.2 for its dielectric anisotropy. This value is very small so the needed electric fields must be high if a strong electrooptic effect is desired. Hence, further work with other compounds is recommended.

\section{References}

1. H. Stegemeyer and K. Bergmann, "Experimental Results and Problems Concerning Blue Phases," Springer Series Chem. Phys. 11, $161(1980)$.

2. O. Lehmann, "Stoffe mit drei flussigen Zustanden, einem isotropund zwei kristallinisch-flussigen," Z. Phys. Chem. 56, 750 (1906).

3. S. A. Brazovskii, "Phase Transition of an Isotropic System to a Nonuniform State," Sov. Phys. JETP 41, 85 (1975).

4. S. Meiboom, M. Sammon, and W. F. Brinkman, "A Lattice of Disclinations: the Structure of the Blue Phases of Cholesteric Liquid Crystals," Phys. Rev. A 27, 438 (1983).

5. H. Grebel, R. M. Hornreich, and S. Shtrikman, "Landau Theory of Cholesteric Blue Phases," Phys. Rev. A 28, 1114 (1983).

6. K. Bergmann and H. Stegemeyer, "Evidence for Polymorphism Within the So-Called Blue Phase of Cholesteric Esters. IV. Temperature and Angular Dependence of Selective Reflection," Z. Naturforsch. Teil A 34, 1031 (1979).

7. D. L. Johnson, J. H. Flack, and P. P. Crooker, "Structure and Properties of the Cholesteric Blue Phases," Phys. Rev. Lett. 45, 641 (1980).

8. P. G. de Gennes, The Physics of Liquid Crystals (Clarendon, Oxford, 1974).

9. D. Armitage and F. P. Price, "Calorimetry of Liquid Crystal Phase Transitions,” J. Phys. París 36, C1-133 (1975).

10. B. M. León Fong, J. A. Martín-Pereda, G. Heppke, H. S. Kitzerow, and F. Oestreicher, "Phasendiagramme einiger chiral-racemischer 'Blue Phase'-Systeme," communication presented at the Twenty-First Bunsen-Colloquium on Material Properties and Order States in Liquid crystals, Berlin (Sept. 1983).

11. P. L. Finn and P. E. Cladis, "Cholestric Blue Phases in Mixtures and in an Electric Field," Mol. Cryst. Liq. Cryst. 84, 159 (1982).

The authors wish to thank G. Heppke for the use of his laboratory during part of this work and for helpful discussions.

$\begin{array}{ll}\text { name of conference: } & \text { OPTICS } 186 \\ \text { sponsored by } & \text { Deutsche Gesellschaft für } \\ & \text { angewandte Optik; } \\ & \text { Optical Group, the Institute } \\ & \text { of Physics (UK); } \\ & \text { Netherlands Optical Commission } \\ & : \text { May } 21-24,1986 \\ \text { date } & : \text { Scheveningen, the Netherlands } \\ \text { location } & \text { Oldelft.F. de Meijere } \\ \text { contact } & \text { P.O.Box } 72 \\ & 2600 \text { MD Delft, the Netherlands } \\ & \text { phone: } 015-601901\end{array}$

